\title{
Principal properties (PPs) as solvent descriptors for multivariate optimization in organic synthesis: specific PPs for ethers
}

\author{
Francesco P. Ballistreri, Cosimo G. Fortuna, Giuseppe Musumarra,* Didier Pavone, \\ and Salvatore Scirè
}

\author{
Dipartimento di Scienze Chimiche, Università di Catania, \\ Viale Andrea Doria 6, 95125 Catania, Italy \\ E-mail: gmusumarra@dipchi.unict.it
}

Dedicated to Professor Domenico Spinelli on his $70^{\text {th }}$ birthday

(received 07 Oct 02; accepted 21 Nov 02; published on the web 29 Nov 02)

\begin{abstract}
Principal properties (PPs) for solvents were extended to 113 solvents by the addition of ten ethers. Specific "PPs" for ethers, suitable for solvent optimization in Grignard reactions, were also derived and their physico-chemical significance discussed.
\end{abstract}

Keywords: Solvent descriptors, principal properties, Grignard reaction

\section{Introduction}

The application of multivariate strategies provides great advantages in optimizing desired properties (yield, regio- or stereoisomeric ratios, etc.) by simultaneous variation of experimental conditions - which may be continuous (temperature, time, concentrations, etc.) and discrete (solvent, catalyst, etc.) variables: the latter are parameterized by the so-called principal properties (PPs). Intrinsic properties suitable for experimental design need to be orthogonal to each other. In order to fulfil the above requirement, the concept of principal properties (PPs), i.e., of intrinsic properties representative of experimentally observable macroscopic descriptors, has recently been introduced. ${ }^{1}$

PPs, calculated as the principal component analysis (PCA) scores from several experimentally measured properties, are now available for solvents ${ }^{1}$, aldehydes and ketones, ${ }^{1}$ amines, ${ }^{1}$ Lewis acids, ${ }^{1}$ aromatic substituents ${ }^{2}$ and amino acids. ${ }^{3-5}$ First and second generation principal properties for heteroaromatics moieties, based on aromaticity ${ }^{6}$ and on 3D-GRID structural parameters ${ }^{7}$ respectively, have been reported by our group.

A typical example of the potentialities of optimization in PPs is provided by the results of a multivariate experimental design, based on the PPs of Lewis acid catalysts and solvents, which 
give a better understanding of the effects of the above parameters on the isomeric distribution in the reaction of phenylhydrazones derived from unsymmetrical ketones (the well known Fischer indole synthesis). Multivariate optimization achieved not only regiospecific synthesis of single indole regioisomers, ${ }^{8}$ but almost quantitative yields in a single step (one-pot) reaction. ${ }^{9}$ It is striking that the above result was achieved for a reaction, such as the Fischer indole synthesis ${ }^{10}$ known for more than a century, and to which an entire book has been devoted. ${ }^{11}$

In addition to statistical orthogonality, PPs derived from PCA multivariate characterizations have the advantage of being less influenced by measurement errors and system-specific variations than are single descriptors. Moreover, reliable PPs can be obtained from original data matrices even with missing data.

However, as PPs are derived by an empirical statistical method, such as PCA, carried out on a data matrix with a given number of objects and variables, the updating of such descriptors is needed as new properties (variables) become available for "old" and "new" objects. In particular, solvent PPs first derived by Carlson and co-workers based on eight parameters for 82 solvents $^{12}$ have been extended by the same author ${ }^{1}$ to 103 solvents and integrated by addition of a $9^{\text {th }}$ variable (water-solubility).

For specific organic syntheses such as Grignard reactions, however, the use of solvent is chemically limited to a specific class, i.e., ethers.

In this context, we extend the solvent set by addition of ten solvents, all ethers, to the 103 considered by Carlson ${ }^{1}$ in order to derive PPs from a larger data set containing 113 solvents.

Furthermore, we also consider an "ether" class model in order to derive "ether PPs" which might be more informative in characterizing the above solvents specifically for the Grignard reaction.

\section{Results and Discussion}

Table 1 reports the data set considered to derive PPs in ref. 1, integrated by corrected or new descriptors for the original 103 solvents and expanded by including descriptors for ten new solvents (all ethers). PCA of the above data matrix, including 113 solvents and 9 descriptors (cf. Table 1) provided a 2- principal-components (PC) model explaining $69.4 \%$ of the variance. The scores of such a model, new PPs for all 113 solvents, are also recorded in Table 1. 
Table 1. Descriptors (1-9) and principal properties $\left(t_{1}\right.$ and $\left.t_{2}\right)$ for solvents $1-113^{a}$

\begin{tabular}{|c|c|c|c|c|c|c|c|c|c|c|c|c|c|}
\hline \multirow[t]{2}{*}{ Entry } & \multirow[t]{2}{*}{$\mathrm{CAS}^{\mathrm{b}}$} & \multirow[t]{2}{*}{ Solvent } & \multicolumn{9}{|c|}{ Descriptors } & \multicolumn{2}{|c|}{ PPs } \\
\hline & & & 1 & 2 & 3 & 4 & 5 & 6 & 7 & 8 & 9 & $\mathrm{t}_{1}$ & $\mathrm{t}_{2}$ \\
\hline 1 & $7732-18-5$ & Water & 0 & 100 & 78.3 & 5.9 & 1.333 & 1 & 0.9282 & -1.38 & 1.774 & 3.67 & -1.78 \\
\hline 2 & $75-12-7$ & Formamide & 2.55 & 210.5 & 111 & 11.2 & 1.4475 & 0.8 & 1.159 & -1.51 & 1.401 & 4.56 & 0.67 \\
\hline 3 & $107-21-1$ & 1,2-Ethanediol & -12.6 & 197.15 & 37.7 & 7.7 & 1.4318 & 0.79 & 1.1088 & -1.36 & 1.06 & 2.93 & 0.16 \\
\hline 4 & $67-56-1$ & Methanol & -97.7 & 64.5 & 32.66 & 5.5 & 1.3284 & 0.76 & 0.7914 & -0.77 & 1.393 & 1.63 & -3.04 \\
\hline 5 & $123-39-7$ & N-Methylformamide & -3.8 & 182.5 & 182.4 & 12.9 & 1.4319 & 0.72 & 1.01 & -0.6 & & 5.84 & -0.13 \\
\hline 6 & $111-46-6$ & Diethylene glycol & -7.8 & 245.7 & 31.69 & 7.7 & 1.4475 & 0.71 & 1.109 & -1.51 & & 3.06 & 0.65 \\
\hline 7 & $112-27-6$ & Triethylene glycol & -4.3 & 288 & 23.69 & 10 & 1.4558 & 0.7 & 1.125 & -1.33 & 0.852 & 3.07 & 1.21 \\
\hline 8 & $109-86-4$ & 2-Methoxyethanol & -85.1 & 124.6 & 16.93 & 6.8 & 1.4021 & 0.67 & 0.965 & -0.77 & 1.103 & 1.54 & -1.45 \\
\hline 9 & $79-16-3$ & N-Methylacetamide & 30.6 & 206.7 & 191.3 & 14.2 & 1.4253 & 0.67 & 0.957 & -1.05 & & 6.48 & 0.15 \\
\hline 10 & $64-17-5$ & Ethanol & -114.5 & 78.3 & 24.55 & 5.8 & 1.3614 & 0.65 & 0.785 & -0.31 & 1.231 & 1.11 & -2.71 \\
\hline 11 & $141-43-5$ & 2-Aminoethanol & 10.5 & 170.95 & 37.72 & 7.6 & 1.4545 & 0.65 & 1.018 & -1.31 & 1.216 & 2.66 & 0.27 \\
\hline 12 & $64-19-7$ & Acetic acid & 16.7 & 117.9 & 6.17 & 5.6 & 1.3719 & 0.65 & 1.0492 & -0.17 & 1.002 & 1.56 & -0.54 \\
\hline 13 & $100-51-6$ & Benzyl alcohol & -15.3 & 205.45 & 13.1 & 5.5 & 1.5404 & 0.61 & 1.042 & 1.1 & -0.992 & 0.71 & 1.67 \\
\hline 14 & $71-23-8$ & 1-Propanol & -126.2 & 97.15 & 20.45 & 5.5 & 1.3856 & 0.62 & 0.804 & 0.25 & 1.126 & 0.78 & -2.35 \\
\hline 15 & $71-36-3$ & 1-Butanol & -88.6 & 117.7 & 17.51 & 5.8 & 1.3993 & 0.6 & 0.8098 & 0.88 & 0.125 & 0.52 & -1.47 \\
\hline 16 & $78-83-1$ & 2-Methyl-1-propanol & -108 & 107.9 & 17.93 & 6 & 1.3959 & 0.55 & 0.794 & 0.76 & 0.176 & 0.36 & -1.78 \\
\hline 17 & $67-63-0$ & 2-Propanol & -88 & 82.2 & 19.92 & 5.5 & 1.3772 & 0.55 & 0.786 & 0.05 & 1.117 & 0.81 & -2.19 \\
\hline 18 & $78-92-2$ & 2-Butanol & -114.7 & 99.5 & 16.56 & 5.5 & 1.3971 & 0.51 & 0.808 & 0.61 & 0.176 & 0.19 & -1.87 \\
\hline 19 & $123-51-3$ & 3-Methyl-1-butanol & -117.2 & 130.5 & 15.19 & 6.1 & 1.4072 & 0.57 & 0.8092 & 1.22 & -0.518 & 0.07 & -1.42 \\
\hline 20 & $108-93-0$ & Cyclohexanol & 25.25 & 161.1 & 15 & 6.2 & 1.4648 & 0.5 & 0.9642 & 1.23 & -0.755 & 0.68 & 1.01 \\
\hline 21 & $4427-89-8$ & 4-Methyl-1,3-dioxol-2-one & -54.5 & 241.7 & 64.94 & 16.5 & 1.4215 & 0.49 & 1.204 & 0.86 & & 3.14 & 0.76 \\
\hline 22 & $6032-29-7$ & 2-Pentanol & -73 & 119 & 13.71 & 5.5 & 1.4064 & 0.49 & 0.81 & 1.19 & -0.612 & -0.01 & -1.05 \\
\hline 23 & $75-52-5$ & Nitromethane & -28.55 & 101.2 & 35.94 & 11.9 & 1.3819 & 0.48 & 1.137 & -0.35 & 0.235 & 1.80 & -0.52 \\
\hline 24 & $75-05-8$ & Acetonitrile & -43.8 & 81.6 & 35.94 & 11.8 & 1.3441 & 0.46 & 0.7857 & -0.34 & 1.282 & 1.85 & -1.94 \\
\hline 25 & $584-02-1$ & 3-Pentanol & -75 & 115.3 & 13.35 & 5.5 & 1.4104 & 0.46 & 0.821 & 1.21 & -0.234 & 0.02 & -1.08 \\
\hline 26 & $67-68-5$ & Dimethyl sulfoxide & 18.5 & 189 & 46.45 & 13.5 & 1.4793 & 0.44 & 1.101 & -1.35 & & 3.19 & 1.06 \\
\hline 27 & $62-53-3$ & Aniline & -6 & 184.4 & 6.71 & 5 & 1.5863 & 0.42 & 1.0217 & 0.9 & -0.37 & 0.39 & 1.91 \\
\hline 28 & $126-33-0$ & Sulfolane & 28.45 & 287.3 & 43.3 & 16 & 1.4816 & 0.41 & 1.262 & -0.77 & & 3.74 & 2.31 \\
\hline 29 & $108-24-7$ & Acetic anhydride & -73.1 & 140 & 20.7 & 9.4 & 1.3904 & 0.41 & 1.082 & -0.48 & 0.07 & 1.09 & -0.74 \\
\hline 30 & $75-65-0$ & 2-Methyl-2-propanol & 25.6 & 82.3 & 12.47 & 5.5 & 1.3877 & 0.39 & 0.789 & 0.35 & -1.057 & 0.19 & -0.55 \\
\hline 31 & $68-12-2$ & N,N-dimethylformamide & -60.4 & 153 & 36.71 & 10.8 & 1.4305 & 0.4 & 0.945 & -1.01 & 1.112 & 1.94 & -0.65 \\
\hline 32 & $127-19-5$ & $\mathrm{~N}, \mathrm{~N}$-dimethylacetamide & -20 & 166.1 & 37.78 & 12.4 & 1.4384 & 0.38 & 0.937 & -0.77 & 1.11 & 2.23 & -0.04 \\
\hline 33 & $107-12-0$ & Cyanopropane & -92.8 & 97.35 & 28.86 & 11.7 & 1.3658 & 0.4 & 0.782 & 0.16 & & 0.87 & -1.96 \\
\hline 34 & $872-50-4$ & 1-Methyl-pyrrolidine-2-one & -24.4 & 202 & 32.2 & 13.6 & 1.47 & 0.36 & 1.026 & -0.39 & & 2.17 & 0.78 \\
\hline 35 & $67-64-1$ & Acetone & -94.7 & 56.1 & 20.56 & 9 & 1.3587 & 0.36 & 0.79 & -0.24 & 1.134 & 0.74 & -2.46 \\
\hline 36 & $98-95-3$ & Nitrobenzene & 5.8 & 210.8 & 34.78 & 13.3 & 1.5562 & 0.32 & 1.204 & 1.85 & -1.778 & 0.92 & 2.88 \\
\hline 37 & $100-47-0$ & Benzonitrile & -12.75 & 191.1 & 25.2 & 13.4 & 1.5282 & 0.33 & 1.01 & 1.56 & -1.013 & 0.93 & 1.79 \\
\hline
\end{tabular}


Table 1. Continued

\begin{tabular}{|c|c|c|c|c|c|c|c|c|c|c|c|c|c|}
\hline \multirow[t]{2}{*}{ Entry } & \multirow[t]{2}{*}{$\mathrm{CAS}^{\mathrm{b}}$} & \multirow[t]{2}{*}{ Solvent } & \multicolumn{9}{|c|}{ Descriptors } & \multicolumn{2}{|c|}{ PPs } \\
\hline & & & 1 & 2 & 3 & 4 & 5 & 6 & 7 & 8 & 9 & $t_{1}$ & $t_{2}$ \\
\hline 38 & $107-15-3$ & 1,2-Diaminoethane & 11.3 & 116.9 & 12.9 & 6.3 & 1.4568 & 0.35 & 0.899 & -2.04 & & 1.22 & -0.14 \\
\hline 39 & $107-06-2$ & 1,2-Dichloroethane & -35.7 & 83.5 & 10.7 & 6.1 & 1.4448 & 0.33 & 1.235 & 1.48 & -1.092 & -0.38 & 0.40 \\
\hline 40 & $75-85-4$ & 2-Methyl-2-butanol & -8.8 & 102 & 5.78 & 5.7 & 1.405 & 0.32 & 0.806 & 0.89 & 0.039 & 0.09 & -0.64 \\
\hline 41 & $78-93-3$ & 2-Butanone & -86.7 & 79.6 & 18.51 & 9.2 & 1.3788 & 0.33 & 0.805 & 0.29 & 0.494 & 0.46 & -1.84 \\
\hline 42 & $98-86-2$ & Acetophenone & 19.6 & 202 & 17.39 & 9.8 & 1.5342 & 0.31 & 1.0281 & 1.58 & -1.553 & 0.44 & 2.28 \\
\hline 43 & $75-09-2$ & Dichloromethane & -94.9 & 39.6 & 8.93 & 5.2 & 1.4242 & 0.31 & 1.33 & 1.25 & -0.699 & -0.76 & -0.57 \\
\hline 44 & $632-22-4$ & 1,1,3,3-Tetramethylurea & -1.2 & 175.2 & 23.6 & 11.7 & 1.4493 & 0.32 & 0.969 & 0.19 & & 1.50 & 0.66 \\
\hline 45 & $680-31-9$ & Hexamethylphosphoramide & 7.2 & 233 & 29.6 & 18.5 & 1.4588 & 0.32 & 1.024 & 0.28 & & 2.76 & 1.38 \\
\hline 46 & $108-94-1$ & Cyclohexanone & -32.1 & 155.65 & 16.1 & 10.3 & 1.451 & 0.28 & 0.9478 & 0.81 & -1.236 & 0.32 & 0.47 \\
\hline 47 & $110-86-1$ & Pyridine & -41.55 & 115.25 & 12.91 & 7.9 & 1.5102 & 0.3 & 0.982 & 0.65 & 1.094 & 0.64 & 0.22 \\
\hline 48 & $79-20-9$ & Methyl acetate & -98.05 & 56.9 & 6.68 & 5.7 & 1.3614 & 0.25 & 0.933 & 0.18 & 0.486 & -0.25 & -2.07 \\
\hline 49 & $108-10-1$ & 4-Methyl-2-pentanone & -84.7 & 117.4 & 13.11 & 2.7 & 1.3958 & 0.27 & 0.7978 & 1.31 & -0.871 & -0.90 & -1.19 \\
\hline 50 & $75-34-3$ & 1,1-Dichloroethane & -97 & 57.3 & 10 & 6.1 & 1.4164 & 0.27 & 1.176 & 1.79 & -1.259 & -1.03 & -0.63 \\
\hline 51 & $91-22-5$ & Quinoline & -14.85 & 237.1 & 8.95 & 7.3 & 1.6273 & 0.27 & 1.093 & 2.03 & & 0.09 & 3.21 \\
\hline 52 & $96-22-0$ & 3-Pentanone & -39 & 102 & 17 & 9.4 & 1.3923 & 0.27 & 0.8138 & 0.91 & -0.27 & 0.25 & -0.86 \\
\hline 53 & $67-66-3$ & Chloroform & -63.15 & 61.2 & 4.81 & 3.8 & 1.4459 & 0.26 & 1.48 & 1.97 & -1.246 & -1.14 & 0.52 \\
\hline 51 & $11210 ?$ & Triclermo & 15 & 216 & 75 & & 11761 & $0 \cap 5$ & ก ก & 115 & & ก กо & 015 \\
\hline 55 & $111-96-6$ & Diglyme & -64 & 162 & 7.23 & 6.6 & 1.4078 & 0.24 & 0.945 & -0.80 & & 0.23 & -0.59 \\
\hline 56 & $110-71-4$ & Glyme & -69 & 85 & 7.2 & 5.7 & 1.3796 & 0.23 & 0.8269 & -0.21 & & -0.49 & -1.53 \\
\hline 57 & $95-50-1$ & 1,2-Dichlorobenzene & -17 & 180.5 & 9.93 & 7.1 & 1.5515 & 0.23 & 1.305 & 3.38 & -3.029 & -1.18 & 2.90 \\
\hline 58 & $141-78-6$ & Ethyl acetate & -83.55 & 77.1 & 6.02 & 6.1 & 1.3724 & 0.23 & 0.9 & 0.73 & -0.041 & -0.44 & -1.59 \\
\hline 59 & $462-06-6$ & Fluorobenzene & -42.2 & 84.7 & 5.42 & 4.9 & 1.4684 & 0.19 & 1.023 & 2.27 & -1.797 & -1.35 & 0.39 \\
\hline 60 & $591-50-4$ & Iodobenzene & -31.35 & 188.3 & 4.49 & 4.7 & 1.62 & 0.17 & 1.831 & 3.25 & -2.847 & -1.43 & 4.32 \\
\hline 61 & $108-90-7$ & Chlorobenzene & -45.6 & 131.7 & 5.62 & 5.4 & 1.5248 & 0.19 & 1.106 & 2.84 & -2.45 & -1.51 & 1.51 \\
\hline 62 & $108-86-1$ & Bromobenzene & -30.6 & 155.9 & 5.4 & 5.2 & 1.559 & 0.18 & 1.495 & 2.99 & -2.57 & -1.37 & 2.88 \\
\hline 63 & 109-99-9 & Tetrahydrofuran & -108.4 & 66 & 7.58 & 5.8 & 1.4072 & 0.21 & 0.889 & 0.46 & 1.142 & -0.24 & -1.85 \\
\hline 64 & $100-66-3$ & Methoxybenzene & -37.5 & 153.6 & 4.33 & 4.2 & 1.517 & 0.2 & 0.996 & 2.11 & -1.917 & -1.19 & 1.24 \\
\hline 65 & $103-73-1$ & Ethoxybenzene & -29.5 & 169.8 & 4.22 & 4.5 & 1.5074 & 0.18 & 0.967 & 2.51 & -2.332 & -1.33 & 1.43 \\
\hline 66 & $71-55-6$ & 1,1,1-Trichloroethane & -30.4 & 74.1 & 7.25 & 5.7 & 1.438 & 0.17 & 1.339 & 2.49 & -2.179 & -1.35 & 0.89 \\
\hline 67 & $123-91-1$ & 1,4-Dioxane & 11.8 & 101.3 & 2.21 & 1.5 & 1.4224 & 0.16 & 1.034 & -0.27 & 1.07 & 0.09 & -0.21 \\
\hline 68 & $79-01-6$ & Trichloroethene & -86.4 & 87.2 & 3.42 & 2.7 & 1.4773 & 0.16 & 1.464 & 2.42 & -1.995 & -1.84 & 0.91 \\
\hline 69 & $110-89-4$ & Piperidine & -10.5 & 106.7 & 5.8 & 4 & 1.4525 & 0.15 & 0.861 & 0.85 & & -0.85 & 0.16 \\
\hline 70 & $101-84-8$ & Diphenyl ether & 26.9 & 258.1 & 3.69 & 3.9 & 1.5763 & 0.14 & 1.075 & 4.21 & -3.947 & -1.81 & 3.85 \\
\hline 71 & $60-29-7$ & Diethyl ether & -116.3 & 34.4 & 4.2 & 3.8 & 1.3524 & 0.12 & 0.714 & 0.89 & 0.076 & -1.27 & -2.71 \\
\hline 72 & $71-43-2$ & Benzene & 5.5 & 80.1 & 2.27 & 0 & 1.5011 & 0.11 & 0.8786 & 2.13 & -1.559 & -1.76 & 0.69 \\
\hline 73 & $108-20-3$ & Di-isopropyl ether & -85.5 & 68.3 & 3.88 & 4.2 & 1.3681 & 0.1 & 0.7241 & 1.52 & -0.198 & -1.24 & -1.89 \\
\hline 74 & $108-88-3$ & Toluene & -95 & 110.6 & 2.38 & 1 & 1.4969 & 0.1 & 0.867 & 2.73 & -1.747 & -2.28 & 0.01 \\
\hline
\end{tabular}


Table 1. Continued

\begin{tabular}{|c|c|c|c|c|c|c|c|c|c|c|c|c|c|}
\hline \multirow[t]{2}{*}{ Entry } & \multirow[t]{2}{*}{$\mathrm{CAS}^{\mathrm{b}}$} & \multirow[t]{2}{*}{ Solvent } & \multicolumn{9}{|c|}{ Descriptors } & \multicolumn{2}{|c|}{ PPs } \\
\hline & & & 1 & 2 & 3 & 4 & 5 & 6 & 7 & 8 & 9 & $t_{1}$ & $t_{2}$ \\
\hline 75 & $142-96-1$ & Dibutyl ether & -95.2 & 140.3 & 3.08 & 3.9 & 1.3992 & 0.07 & 0.7689 & 3.21 & -1.941 & -2.10 & -0.64 \\
\hline 76 & $121-44-8$ & Triethylamine & -114.7 & 88.9 & 2.42 & 2.9 & 1.401 & 0.04 & 0.7275 & 1.45 & 0.224 & -1.44 & -1.83 \\
\hline 77 & $108-67-8$ & 1,3,5-Trimethylbenzene & -44.7 & 164.7 & 2.28 & 0 & 1.4994 & 0.07 & 0.865 & 3.42 & -3.356 & -2.68 & 1.22 \\
\hline 78 & $75-15-0$ & Carbon disulfide & -111.6 & 46.2 & 2.64 & 0 & 1.6225 & 0.07 & 1.263 & 1.94 & -1.552 & -2.43 & 1.09 \\
\hline 79 & $56-23-5$ & Carbon tetrachloride & -22.8 & 76.6 & 2.23 & 0 & 1.4602 & 0.05 & 1.59 & 2.83 & -2.294 & -2.22 & 1.63 \\
\hline 80 & $127-18-4$ & Tetrachloroethene & -22.4 & 121.2 & 2.3 & 0 & 1.5057 & 0.04 & 1.623 & 3.4 & -2.895 & -2.42 & 2.56 \\
\hline 81 & $110-82-7$ & Cyclohexane & 6.7 & 80.7 & 2.02 & 0 & 1.4262 & 0.01 & 0.778 & 3.44 & -3.069 & -2.76 & 0.34 \\
\hline 82 & $110-54-3$ & Hexane & -95.3 & 68.7 & 1.88 & 0 & 1.3749 & 0.01 & 0.66 & 3.98 & -3.359 & -3.53 & -1.26 \\
\hline 83 & $112-60-7$ & Tetraethylene glycol & -6.2 & 327.3 & 19.7 & 10.8 & 1.4577 & 0.66 & 1.1285 & -2.23 & & 3.74 & 1.29 \\
\hline 84 & $71-41-0$ & 1-Pentanol & -78.2 & 138 & 13.9 & 5.7 & 1.41 & 0.59 & 0.8144 & 1.56 & -0.631 & 0.15 & -0.92 \\
\hline 85 & $80-73-9$ & 1,3-Dimethylethyleneurea & 8.2 & 225.5 & 37.6 & 13.6 & 1.4707 & 0.36 & 1.052 & -0.02 & & 2.49 & 1.36 \\
\hline 86 & $7226-23-5$ & 1,3-Dimethylpropyleneurea & -20 & 230 & 36.12 & 14.1 & 1.4881 & 0.35 & & 0.36 & & 2.18 & 1.54 \\
\hline 87 & $107-87-9$ & 2-Pentanone & -76.9 & 102.3 & 15.38 & 9 & 1.3908 & 0.32 & 0.8089 & 0.91 & -0.328 & 0.10 & -1.27 \\
\hline 88 & 110-91-8 & Morpholine & -4.8 & 128.9 & 7.42 & 5.2 & 1.4542 & 0.31 & 1.0005 & -0.86 & 1.06 & 0.95 & -0.04 \\
\hline 89 & $563-80-4$ & 3-Methyl-2-butanone & -92 & 94.2 & 15.87 & 9.2 & 1.388 & 0.32 & 0.8051 & 0.56 & -0.164 & 0.15 & -1.57 \\
\hline 90 & $75-97-8$ & 3,3-Dimethyl-2-butanone & -49.8 & 106.3 & 13.1 & 9.3 & 1.3952 & 0.26 & 0.8012 & 1.07 & -0.706 & -0.05 & -0.84 \\
\hline 91 & $565-80-0$ & 2,4-Dimethyl-3-pentanone & -69 & 125.25 & 17.2 & 9.1 & 1.3999 & 0.25 & 0.8108 & 1.49 & -1.299 & -0.34 & -0.69 \\
\hline 92 & $108-83-8$ & 2,6-dimethyl-4-heptanone & -46 & 168.2 & 9.91 & 8.9 & 1.4122 & 0.23 & 0.8053 & 2.96 & -1.731 & -0.75 & 0.18 \\
\hline 93 & $112-36-7$ & Diethyleneglycol diethyl ether & -44.3 & 188.9 & 5.7 & & 1.4115 & 0.21 & 0.9063 & 0.26 & 0.076 & 0.34 & -0.16 \\
\hline 94 & $105-58-8$ & Diethyl carbonate & -43 & 126.8 & 2.82 & 3 & 1.3837 & 0.19 & 0.9752 & 1.21 & -0.798 & -0.84 & -0.49 \\
\hline 95 & $75-35-4$ & 1,1-Dichloroethene & -122 & 31.6 & 4.82 & 4.3 & 1.4247 & 0.19 & 1.218 & 1.86 & -1.666 & -1.77 & -0.83 \\
\hline 96 & 1634-04-4 & tert-Butyl methyl ether & -108.6 & 55.2 & 4.5 & 4.1 & 1.369 & 0.12 & 0.7405 & 0.94 & & -1.81 & -2.09 \\
\hline 97 & $109-89-7$ & Diethylamine & -49.8 & 55.55 & 3.78 & 4 & 1.3846 & 0.15 & 0.7056 & 0.58 & & -1.31 & -1.53 \\
\hline 98 & $111-43-3$ & Dipropyl ether & -123.2 & 90.1 & 3.39 & 4.4 & 1.3805 & 0.1 & 0.736 & 2.03 & -1.388 & -1.83 & -1.72 \\
\hline 99 & $106-42-3$ & 1,4-Dimethylbenzene & 13.3 & 138.4 & 2.27 & 0 & 1.4958 & 0.07 & 0.8611 & 3.15 & -2.806 & -2.24 & 1.44 \\
\hline 100 & $102-82-9$ & Tributylamine & -70 & 214 & & 2.6 & 1.4291 & 0.04 & 0.7771 & 4.56 & -3.116 & -2.84 & 0.72 \\
\hline 101 & 493-01-6 & cis-Decalin & -43 & 195.8 & 2.2 & 0 & 1.481 & 0.02 & 0.8965 & 5.08 & -5.192 & -3.64 & 1.89 \\
\hline 102 & $142-82-5$ & $n$-Heptane & -90.6 & 98.4 & 1.92 & 0 & 1.3876 & 0.01 & 0.6838 & 4.57 & -4.046 & -3.75 & -0.67 \\
\hline 103 & $109-66-0$ & $n$-Pentane & -129.7 & 36.1 & 1.84 & 0 & 1.3575 & 0.01 & 0.6262 & 3.39 & -3.129 & -3.59 & -2.14 \\
\hline 104 & $142-68-7$ & Tetrahydropyran & -49.2 & 88 & 5.66 & 5.8 & 1.4211 & 0.17 & 0.8814 & 0.82 & -0.031 & -0.44 & -0.77 \\
\hline 105 & $693-65-2$ & Dipentyl ether & -69.43 & 186.75 & 2.8 & & 1.412 & & 0.7833 & 4.17 & & -2.23 & 0.26 \\
\hline 106 & $544-01-4$ & Di-isopentyl ether & & 172 & 2.82 & & 1.408 & & 0.775 & 3.8 & & -2.12 & 0.05 \\
\hline 107 & $93-18-5$ & 2-Ethoxynaphthalene & 37.5 & 280 & & & 1.5932 & & 1.064 & 3.896 & & 0.51 & 3.95 \\
\hline 108 & $994-05-8$ & tert-Amyl methyl ether & -80 & 86.3 & & & 1.3885 & 0.11 & 0.7703 & 1.678 & & -2.01 & -1.22 \\
\hline 109 & $637-92-3$ & tert-Butyl ethyl ether & -94 & 70 & & & 1.376 & 0.12 & 0.7404 & 1.678 & & -1.94 & -1.67 \\
\hline 110 & $91-16-7$ & 1,2-Dimethoxybenzene & 22.5 & 206.5 & & & & 0.24 & 1.084 & 1.955 & & 0.54 & 2.41 \\
\hline 111 & $103-50-4$ & Dibenzyl ether & 3.6 & 296 & 3.82 & & 1.5406 & 0.14 & 1.0014 & 3.948 & & -0.81 & 3.27 \\
\hline
\end{tabular}


Table 1. Continued

\begin{tabular}{|c|c|c|c|c|c|c|c|c|c|c|c|c|}
\hline \multirow[t]{2}{*}{ Entry } & \multirow[t]{2}{*}{$\mathrm{CAS}^{\mathrm{b}}$} & \multirow[t]{2}{*}{ Solvent } & \multicolumn{9}{|c|}{ Descriptors } & PPs \\
\hline & & & 1 & 2 & 3 & 4 & 5 & 6 & 7 & 8 & 9 & $\mathrm{t}_{2}$ \\
\hline 112 & $109-87-5$ & Dimethoxymethane & -105 & 43 & 2.64 & & 1.3541 & 0.16 & & -0.265 & & $-1.26-2.58$ \\
\hline 113 & $462-95-3$ & Diethoxymethane & -66.5 & 88 & 2.53 & & & 0.1 & & 0.797 & & $-1.39-0.82$ \\
\hline
\end{tabular}

(a) Values different from those in ref. 1 are in red, newly inserted data in blue. Descriptors are those reported in ref. 1: 1 , melting point $\left({ }^{\circ} \mathrm{C}\right) ; 2$, boiling point $\left({ }^{\circ} \mathrm{C}\right) ; 3$, dielectric constant; 4 , dipole moment $\times 10^{30}(\mathrm{C} \mathrm{m})$, 5, refractive index; 6, $\mathrm{E}_{\mathrm{T}}$, the normalized Reichardt-Dimroth parameter $(\mathrm{kcal} / \mathrm{mol}) ; 7$, density $\times 10^{3}\left(\mathrm{~kg} / \mathrm{m}^{3}\right)$; 8 , lipophilicity as measured by $\log \mathrm{P}$ where $\mathrm{P}$ is the equilibrium constant of the distribution of the solvent between 1 -octanol and water at $25^{\circ} \mathrm{C}$; 9, water solubility $(\log \mathrm{mol} / \mathrm{L})$.

(b) Chemical Abstracts Registry Number
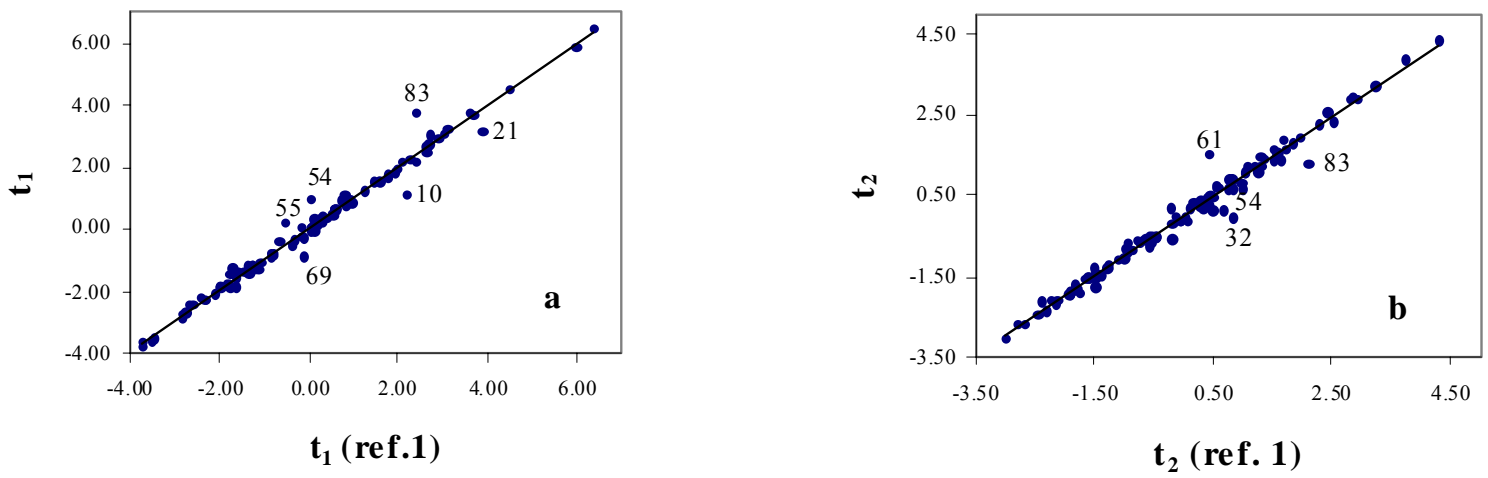

Figure 1. Correlation between PPs for solvents 1-103 derived in the present work and those in ref. 1 .

Figure 1 shows that, as expected, the PPs for solvents 1-103 derived from the 113 solvents model, with a few exceptions (differences above 0.5 are discussed below), closely resemble those of the 103 solvents model reported in ref. 1 . Differences in both $t_{1}$ and $t_{2}$ found for triglyme (54) and tetraethylene glycol (83) can be ascribed to the addition of new values for 54 and correction of $\log \mathrm{P}$ for 83. Significant differences in $t_{1}$ for ethanol (10) and in $t_{2}$ for $N, N-$ dimethylacetamide (32) are due to corrections of descriptor values, while those in $t_{1}$ for 4methyl-1,3-dioxol-2-one (21) and diglyme (55) are the result of the insertion of new values. Differences in $t_{2}$ for chlorobenzene (61) and in $t_{1}$ for piperidine (69) are probably due to printing errors in Table 15A.1 of ref. 1, as the plot in the same book and PPs in ref. 12 are consistent with our values.

Figure 2, the PCA " $\mathrm{p}_{1}-\mathrm{p}_{2}$ loadings plot" (see equation 1 in the Experimental Section), elucidates the descriptors information content, and provides guidance for interpreting the physico-chemical meaning of solvents PPs. The first PC, exhibiting high $\mathrm{p}_{1}$ values for 
descriptors such as dielectric constant (3), dipole moment (4), $\mathrm{E}_{\mathrm{T}}$ (6), and water-solubility (9), and the lowest $\mathrm{p}_{1}$ value for $\log \mathrm{P}(8)$, can be related to the solvent polarity.

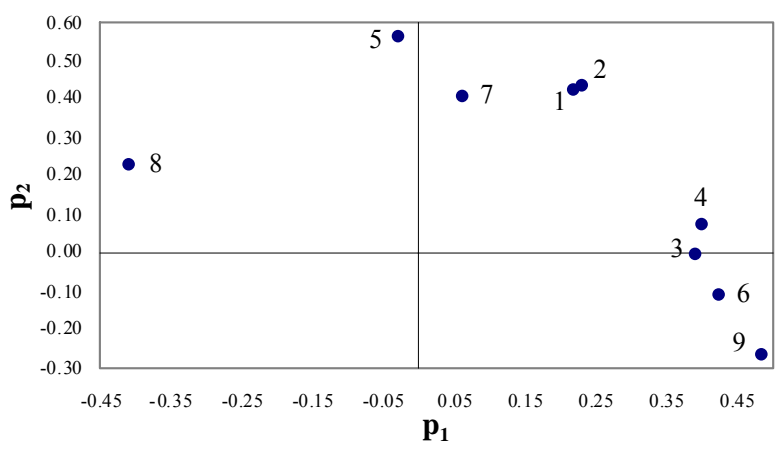

Figure 2. Loadings plot for the 113 solvents model.

Table 2. Principal properties $\left(t_{1}, t_{2}\right.$ and $\left.t_{3}\right)$ for the 24 ethers model

\begin{tabular}{|c|c|c|c|c|}
\hline Entry & Solvents & & PPs & \\
\hline & & $\mathrm{t}_{1}$ & $t_{2}$ & $t_{3}$ \\
\hline 54 & Triglyme & 1.20 & -2.82 & 0.33 \\
\hline 55 & Diglyme & 0.39 & -2.62 & 0.34 \\
\hline 56 & Glyme & -0.73 & -2.32 & 0.45 \\
\hline 63 & Tetrahydrofuran & -0.83 & -2.05 & 0.90 \\
\hline 64 & Methoxybenzene & 1.54 & -0.09 & -0.16 \\
\hline 65 & Ethoxybenzene & 1.50 & 0.30 & -0.03 \\
\hline 67 & 1,4-Dioxane & 0.62 & -0.17 & -2.42 \\
\hline 70 & Diphenyl ether & 3.43 & 1.67 & -0.02 \\
\hline 71 & Diethyl ether & -2.64 & 0.11 & 0.08 \\
\hline 73 & Di-isopropyl ether & -2.01 & 0.70 & 0.02 \\
\hline 75 & Dibutyl ether & -1.28 & 1.97 & 0.54 \\
\hline 93 & Diethyleneglycol diethyl ether & 0.52 & -1.30 & 0.14 \\
\hline 96 & Tert-Butyl methyl ether & -2.18 & 0.01 & 0.18 \\
\hline 98 & Dipropyl ether & -2.07 & 1.03 & 0.39 \\
\hline 104 & Tetrahydropyran & -0.29 & -0.81 & -0.06 \\
\hline 105 & Dipentyl ether & -0.05 & 2.36 & 0.70 \\
\hline 106 & Diisopentyl ether & -0.16 & 2.17 & 0.54 \\
\hline 107 & 2-Ethoxynaphthalene & 4.15 & 1.16 & 0.06 \\
\hline 108 & Tert-Amyl methyl ether & -1.45 & 0.75 & -0.16 \\
\hline 109 & Tert-Butyl ethyl ether & -1.83 & 0.57 & 0.08 \\
\hline 110 & 1,2 Dimethoxybenzene & 3.06 & -0.90 & -0.29 \\
\hline 111 & Dibenzyl ether & 2.89 & 1.63 & 0.46 \\
\hline 112 & Dimethoxymethane & -2.39 & -0.24 & -1.04 \\
\hline 113 & Diethoxymethane & -1.64 & 0.92 & -0.92 \\
\hline
\end{tabular}


Accordingly, very high $\mathrm{t}_{1}$ values are exhibited in Table 1 by amides $(9,5$, and 2$)$ and water (1), and very low $\mathrm{t}_{1}$ values by hydrocarbons $(82,101-103)$. Figure 2 also shows high $\mathrm{p}_{2}$ values for m.p. (1), b.p. (2), refractive index (5), and density (7) descriptors, properties affected by the molecular weight within each class of chemically different solvents. Accordingly, $\mathrm{t}_{2}$ values in Table 1 increase on increasing the molecular weight of hydrocarbons (e.g., 103, 82, 102), of primary alcohols (e.g., 4, 10, 14, 15) and of ethers (e.g., 71, 98, 75, 105).

The PPs in Table 1 can be conveniently adopted as solvent descriptors in multivariate optimization of reactions in which a wide range of solvents may be used. However, severe limitations in solvent selection may occur owing to chemical reasons, e.g., the Grignard reaction can be carried out only in ethers. In this case, where a small portion of the experimental space can be investigated, it appears appropriate to derive PPs from a specific "class" model including only chemically similar solvents. Therefore PCA was carried out on a data matrix including 24 objects (ethers 54-56, 63-65, 67, 70, 71, 73, 75, 93, 96, 98, 104-113) and seven variables (descriptors 1-3, 5-8). Exclusion of descriptors 4 and 9 from the analysis is dictated by the lack of the above data for many solvents (see Table 1). PCA provided a 3-PC model explaining 95.1\% of variance (56.5 first PC, 31.6\% second PC and 7.0\% third PC). Ether PPs $\left(\mathrm{t}_{1}, \mathrm{t}_{2}\right.$ and $\left.\mathrm{t}_{3}\right)$ derived from this model are reported in Table 2 and plotted in Figure 3.
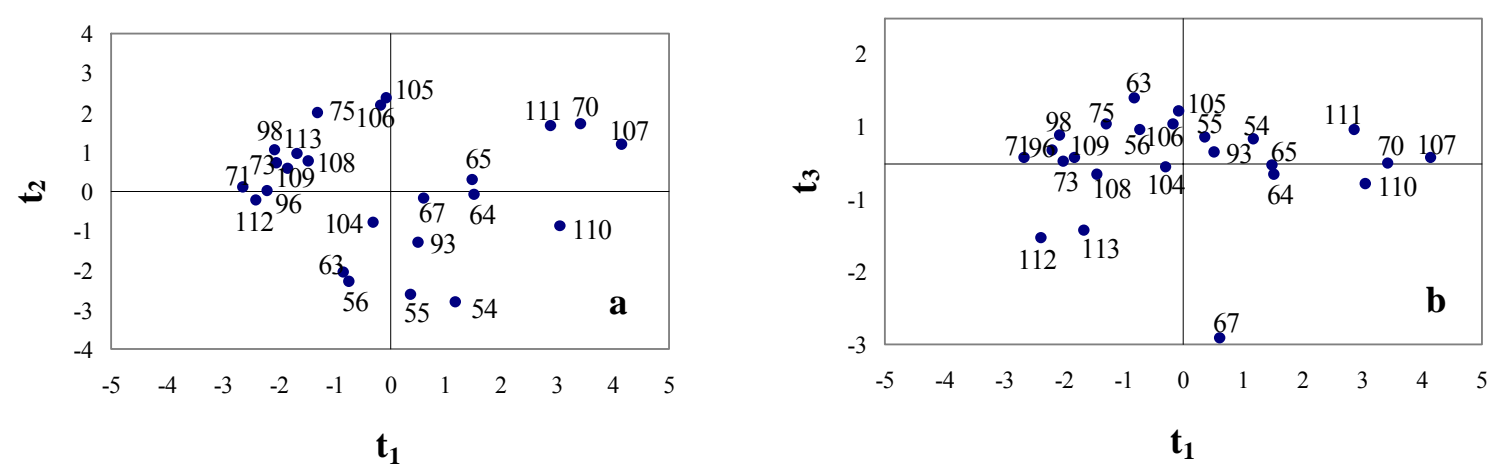

Figure 3. Scores Plots $t_{1}-t_{2}(a)$ and $t_{1}-t_{3}(b)$ for the 24-ethers model.

The first PP ( $t_{1}$ in Table 2$)$, exhibiting an excellent correlation $\left(R^{2}=0.93\right)$ with the second PP derived from the general model ( $\mathrm{t}_{2}$ in Table 1$)$, can be related to the ether molecular weight, i.e., with the lowest value for diethyl ether (71) and very high values for 2-ethoxynaphthalene (107) and diphenyl ether (70). This correlation is not surprising, as by restricting the model to a class of chemically similar solvents, it is expected that molecular weight becomes the first systematic variation evidenced by PCA. The interpretation of the $2^{\text {nd }}$ and $3^{\text {rd }}$ PPs is not straightforward. The loadings plot $\mathrm{p}_{1}-\mathrm{p}_{2}$ shows (Figure 4a) grouping of descriptors 1, 2, 5 and 7 and a clear differentiation of descriptors 3 and $6\left(\right.$ low $\left.\mathrm{p}_{2}\right)$ from 8 (high $\left.\mathrm{p}_{2}\right)$ resembling the trend already observed in the general model (Figure 2), where variable 8 was discriminated from 3 and 6 by the first component. However, the correlation between $t_{2}$ for 24 ethers in the ethers model (Table 
2) and $t_{1}$ in the overall model (Table 1$)$ is very poor $\left(R^{2}=0.53\right)$. This can be reasonably explained by considering that the first component in the general model accounted for, "interclass solvent polarity", which can be roughly represented by the dielectric constant, a bulk property measuring non-specific solvation effects opposite to those of $\log \mathrm{P}$ as, in general, highly polar solvents are not very lipophilic. Accordingly, high $\mathrm{t}_{2}$ values are exhibited in Figure $3 \mathrm{a}$ by symmetrical ethers with a $\mathrm{C}_{4}$ - or $\mathrm{C}_{5^{-}}$chain $(75,105,106)$ and low $\mathrm{t}_{2}$ values by glymes $(54-56)$.
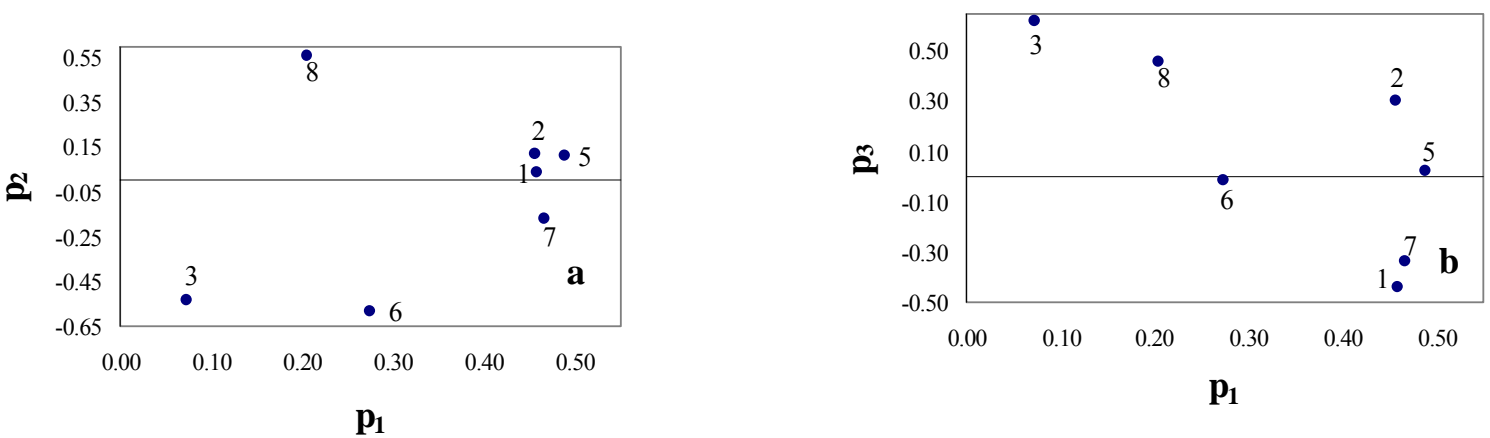

Figure 4. Loadings plot $\mathrm{p}_{1}-\mathrm{p}_{2}(\mathrm{a})$ and $\mathrm{p}_{1}-\mathrm{p}_{3}$ (b) for the 24 ethers models.

The $3^{\text {rd }} \mathrm{PC}$ is required to explain descriptor 3, whose information content, rather than being similar to that of descriptor 6 (see Figure $4 \mathrm{~b}$ ) is closer to that of descriptor 8 , and to differentiate descriptor 2 (boiling point) from descriptor 1 (melting point). In fact, by considering a more homogeneous class, specific solvation effects such as hydrogen bonding and dipole-dipole interactions may be evidenced. Accordingly in Fig. $3 \mathrm{~b}$ the $3^{\text {rd }} \mathrm{PC}$ is required to differentiate solvents with very low $t_{3}$ values such as dioxane (67), dimethoxymethane (112) and diethoxyethane (113), having both low $\varepsilon$ and $\log P$ values (i.e., high water-affinity in spite of their low "bulk" polarity).

\section{Conclusions}

Solvent PPs $\left(t_{1}\right.$ and $\left.t_{2}\right)$ were extended to 113 solvents and specific PPs for ethers $\left(t_{1}, t_{2}\right.$ and $\left.t_{3}\right)$, suitable for solvent optimization in the Grignard reaction, also derived. The score in the ether model $t_{1}$, related to the molecular weight, exhibits an excellent correlation with $t_{2}$ in the overall model, while $t_{2}$ and $t_{3}$ account for specific solvent effects. 


\section{Experimental Section}

General Procedures. The data set used for $\mathrm{PCA}^{13}$ was a table (matrix) in which 113 solvents were characterized by nine physico-chemical properties. The variables have been autoscaled by multiplying the variables by appropriate weights (the reciprocal of the variable standard deviation) to give them unit variance (i.e., the same importance). PCA was carried out by using the SIMCA software package ${ }^{14}$ on a data matrix containing $\mathrm{x}_{\mathrm{ik}}$ elements $(113 \mathrm{x} 9$ for the overall model and $24 \times 7$ for the ethers model, respectively), where the index $k$ is used for the physicochemical properties (variables) and index $i$ for the solvents (objects). Autoscaled matrix elements were then fitted into a model given by Equation (1), where the number A of significant cross terms (components), and the parameters $\mathrm{p}_{\mathrm{ak}}$ and $\mathrm{t}_{\mathrm{ia}}$ are calculated by minimizing the residuals, $\mathrm{e}_{\mathrm{ik}}$, after subtracting $\mathrm{x}_{\mathrm{k}}$ (the mean value of the $\mathrm{i}^{\text {th }}$ experimental quantities $\mathrm{x}_{\mathrm{k}}$ ).

$$
x_{i k}=\bar{x}_{k}+\sum_{a=1}^{a=A} t_{i a} p_{a k}+e_{i k}
$$

Parameters $\overline{\mathrm{x}_{\mathrm{k}}}$ and $\mathrm{p}_{\mathrm{ak}}$ (the loadings) depend only on the physico-chemical properties (variables), and the $t_{i a}$ (scores) only on the solvents.

The deviations from the model are expressed by the residuals, $e_{i k}$. The number of significant components (A) was determined using the cross-validation technique. ${ }^{15}$

\section{Acknowledgments}

We thank the University of Catania for financial support of this work

\section{References}

1. Carlson, R. Design and Optimization in Organic Synthesis; Elsevier: Amsterdam, 1992.

2. Skagerberg, M.; Bonelli, D.; Clementi, S.; Cruciani, G.; Ebert, C. Quant. Struct.-Act. Relat. 1989, 8, 32 .

3. Hellberg, S.; Sjöström, M.; Wold, S. Acta Chem. Scand. 1986, B40, 135.

4. Hellberg, S.; Sjöström, M.; Wold, S. J. Med. Chem. 1987, 30, 1127.

5. Skagerberg, M.; Sjöström, M.; Wold, S. J. Chemometrics 1990, 4, 241.

6. Caruso, L.; Musumarra, G.; Katritzky, A. R. Quant. Struct.-Act. Rel. 1993, 12, 146.

7. Clementi, S.; Cruciani, G.; P. Fifi, P.; Riganelli, D.; Valigi, R.; Musumarra, G. Quant. Struct.-Act. Rel. 1996, 15, 108.

8. Prochazka, M. P.; Carlson, R. Acta Chem. Scand. 1989, 43, 651. 
9. Prochazka, M. P.; Carlson, R. Acta Chem. Scand. 1990, 44, 614.

10. Fischer, E.; Hess, V. F. Ber. Dtsch. Chem. Ges. 1884, 17, 559.

11. Robinson, B. The Fischer Indole Synthesis; Wiley: Chichester, 1982.

12. Carlson, R.; Lundsted, T.; Albano, C. Acta Chem. Scand. 1985, B39, 79.

13. Wold, S. Intell. Lab. Syst. 1987, 2, 37.

14. SIMCA-P 8.0, Umetrics AB, Umea, Sweden, 1999.

15. Wold, S. Technometrics 1978, 20, 397. 